\title{
Resistance of Sclerotinia homoeocarpa Field Isolates to Succinate Dehydrogenase Inhibitor Fungicides
}

James T. Popko, Jr., Stockbridge School of Agriculture, University of Massachusetts, Amherst, MA 01003; Hyunkyu Sang, Department of Plant, Soil, and Microbial Sciences, Michigan State University, East Lansing, MI 48824; Jaemin Lee, Stockbridge School of Agriculture, University of Massachusetts, Amherst, MA 01003; Toshihiko Yamada and Yoichiro Hoshino, Field Science Center for Northern Biosphere, Hokkaido University, Sapporo, Hokkaido 0606-0808, Japan; Geunhwa Jung, ${ }^{\dagger}$ Stockbridge School of Agriculture, University of Massachusetts, Amherst, MA 01003; and Field Science Center for Northern Biosphere, Hokkaido University, Sapporo, Hokkaido 0606-0808, Japan

\begin{abstract}
Sclerotinia homoeocarpa isolates were collected from golf courses in Japan and the United States (2016-2017). Japan isolates were collected during a monitoring study and the U.S. isolates were collected due to field failure. Five succinate dehydrogenase inhibitor (SDHI) active ingredients (boscalid, fluopyram, fluxapyroxad, isofetamid, and penthiopyrad) were examined using in vitro sensitivity assays to determine cross-resistance. Sequence analysis revealed a point mutation leading to an amino acid substitution (H267Y) and a silent mutation (CTT to CTC) at codon 181 in the $S d h$ B subunit gene. Isolates with the BH267Y $(n=10)$ mutation were resistant to boscalid and penthiopyrad

and had increased sensitivity to fluopyram. SdhB silent mutation $181 \mathrm{C}>\mathrm{T}$ isolates $(n=2)$ were resistant to boscalid, isofetamid, and penthiopyrad. Sequence analysis revealed 3 mutations leading to an amino acid substitution (G91R, $n=5$; G150R, $n=1 ; \mathrm{G} 159 \mathrm{~W}, n=1)$ in the $S d h \mathrm{C}$ subunit gene. Isolates harboring the $S d h \mathrm{C}$ (G91R or G150R) mutations were resistant to boscalid, fluxapyroxad, isofetamid, and penthiopyrad. A genetic transformation system was used to generate mutants from a SDHI sensitive isolate to confirm the B-H267Y and C-G91R mutations were direct determinants of SDHI resistance and associated with in vitro sensitivity assay results.
\end{abstract}

Dollar spot, caused by Sclerotinia homoeocarpa (F.T. Bennett), is one of the most common and economically important diseases of cool-season turfgrass species worldwide (Smiley et al. 2005). Cultural practices to manage dollar spot are often ineffective. Therefore, multiple fungicide applications are often needed throughout the growing season to manage dollar spot and maintain high turf quality. However, repeated use of fungicides has led to the development of resistance in $S$. homoeocarpa populations (Smiley et al. 2005). Resistance to the benzimidazole (FRAC \#1) and dicarboximide (FRAC \#2) fungicide classes and reduced sensitivity to the sterol demethylation inhibitor (DMI, FRAC \#3) class have been reported in S. homoeocarpa field populations across North America (Cole et al. 1968; Detweiler et al. 1983; FRAC 2017b; Golembiewski et al. 1995). The succinate dehydrogenase inhibitor (SDHI, FRAC \#7) fungicides have become an important penetrant class for dollar spot control (FRAC 2017b; Vincelli et al. 2017). Currently, five active ingredients (boscalid, fluopyram, fluxapyroxad, isofetamid, and penthiopyrad) are registered for use on turfgrass, and more are in the registration process. Reports of SDHI resistance in other frequently treated plant-pathogenic fungal species have led to heightened alertness for SDHI resistance in S. homoeocarpa due to the heavy dependence on SDHI fungicides for dollar spot control in turfgrass (Sierotzki and Scalliet 2013).

The SDHI fungicide class has expanded rapidly with the discovery of new molecules with broad spectrum fungal control that are very

${ }^{\dagger}$ Corresponding author: G. H. Jung; E-mail: jung@umass.edu

J. T. Popko and H. Sang contributed equally to the manuscript.

Funding: This research is part of fulfillment for Dr. Jung's 6-month sabbatical, which was funded by the Hokkaido University's visiting professor program. This material is based upon work supported by the National Institute of Food and Agriculture, U.S. Department of Agriculture, the Massachusetts Agricultural Experiment Station and the Stockbridge School of Agriculture at the University of Massachusetts Amherst, under project number MAS00436.

Accepted for publication 4 June 2018.

This article is in the public domain and not copyrightable. It may be freely reprinted with customary crediting of the source. The American Phytopathological Society, 2018. useful to the crop protection industry (Sierotzki and Scalliet 2013). Currently, the SDHI class contains 20 different molecules in 10 different chemical groups (FRAC 2017a). SDHI fungicides have a common site-specific mode of action that blocks the succinate dehydrogenase complex composed of four subunits ( $\mathrm{SdhA}, \mathrm{SdhB}, \mathrm{SdhC}$, and $\mathrm{SdhD}$ ) and inhibits mitochondrial electron transport (Matsson and Hederstedt 2001). Succinate dehydrogenase catalyzes the oxidation of succinate to fumarate and the reduction of ubiquinone to quinone, and inhibition leads to decreased energy production and arrested fungal growth (Matsson and Hederstedt 2001). The SDHI class carries a moderate to high resistance risk, which has been reported in 16 different fungal pathogens (FRAC 2017b). The genetic mechanisms responsible for SDHI resistance have been well-studied in both lab and field mutants. Mutations causing resistance in plant pathogenic fungi have been reported in 10 different positions in the $S d h \mathrm{~B}, S d h \mathrm{C}$, and $S d h \mathrm{D}$ genes (FRAC 2017b; Sierotzki and Scalliet 2013). In addition, differential effects of gene mutations on resistance have been reported in Aspergillus oryzae, Botrytis cinerea, Corynespora cassiicola, and Podosphaera xanthii (Ishii et al. 2011; Shima et al. 2011; Veloukas et al. 2013). In $S$. homoeocarpa, anecdotal resistance to SDHIs has been reported since 2004; however, resistance has not been corroborated with in vitro fungicide sensitivity assays or molecular confirmation (Anthony and Kerns 2017; Gilstrap and Vargas 2005).

In this study, $S$. homoeocarpa isolates were initially collected from 12 golf courses in a fungicide resistance monitoring study conducted in Japan. One golf course contained two isolates with reduced boscalid sensitivity collected from a putting green in 2016, and nine additional $S$. homoeocarpa isolates were obtained from a different green at the same Japanese golf course in 2017. While these experiments were being conducted, a golf course in Rhode Island (RI), USA reported (boscalid) field failure, and nine S. homoeocarpa isolates were collected from a fairway in 2017 . In vitro sensitivity and molecular experiments were initiated to compare isolates from the Japanese monitoring study and the RI golf course along with a diverse group of SDHI-sensitive reference isolates. Specific objectives were: 1) to quantify in vitro sensitivities of $S$. homoeocarpa field isolates to boscalid, fluopyram, fluxapyroxad, isofetamid, and penthiopyrad, 2) to sequence the $S d h \mathrm{~B}, S d h \mathrm{C}$, and $S d h \mathrm{D}$ genes of field isolates for potential point mutations associated with SDHI resistance, 3) to understand cross-resistance among five active ingredients mentioned above according to mutation patterns, and 4) to validate the function of point 
mutations in $S d h \mathrm{~B}$ and $S d h \mathrm{C}$ genes using a genetic transformation system.

\section{Materials and Methods}

Origin of fungal isolates. Twenty-five $S$. homoeocarpa field isolates were used in this study. Eleven isolates $(5.1,5.2, \mathrm{~J}-5, \mathrm{~J}-5, \mathrm{~J}-10$, $\mathrm{J}-12, \mathrm{~J}-13$, J-15, J-16, J-17, and J-19) were collected from two putting greens at the Takehara Golf Course (Hiroshima, Japan), which were suspected to be SDHI resistant based on the results of the 2016 in vitro sensitivity monitoring study. Eight isolates (M-1, M-2, M-5, M-7, M-8, M-9, M-10, and M-11) were collected from a golf course fairway in Rhode Island that reported SDHI field efficacy failure and were suspected to be SDHI resistant. Three isolates, 3.1, 6.1, and 6.3, were collected from 2 golf courses in Japan and included because they were sensitive to SDHI fungicides in the 2016 Japanese monitoring study. Isolate JTS30 was sampled from the UMass Turf Research Center (South Deerfield, MA) in an area that had not been exposed to fungicides, and isolate HRS10 was sampled from a golf course in Amherst, MA that had been treated with fungicides, but showed a similar level of fungicide sensitivity to JTS30 (Sang et al. 2015). Isolates JTS30 and HRS10 are sensitive to the DMI, dicarboximide, and SDHI fungicide classes. Isolate CT45 collected from Wethersfield, CT is a dicarboximide-resistant isolate harboring a mutation in the histidine kinase Shos 1 gene and is also resistant to the DMI class (Sang et al. 2017). CT45 represented an isolate that may be found in a golf course setting regularly exposed to fungicides.

SDHI in vitro fungicide sensitivity assays. In vitro sensitivity of $25 \mathrm{~S}$. homoeocarpa isolates to $5 \mathrm{SDHI}$ active ingredients was assayed. $1,000 \mu \mathrm{g} \mathrm{ml}^{-1}$ discriminatory concentrations of the commercial grade fungicides boscalid (Emerald 70WG, BASF), fluopyram (Fluopyram 50SC, Bayer Crop Science), fluxapyroxad (Xzemplar 2.51SC, BASF), isofetamid (Kabuto 3.3SC, PBI Gordon), and penthiopyrad (Velista 50WDG, Syngenta) were used for measuring percentage $(\%)$ of relative mycelium growth (RMG) (Jo et al. 2006). Discriminatory concentrations were selected based on preliminary screening results that showed consistent growth differences among isolates. Discriminatory concentrations were previously used to effectively determine resistance profiles across boscalid, fluopyram, fluxapyroxad, and penthiopyrad in Botrytis cinerea ( $\mathrm{Hu}$ et al. 2016). All isolates were transferred from slant tubes in $-4^{\circ} \mathrm{C}$ and grown on PDA for 3 days at $22^{\circ} \mathrm{C}$. Agar plugs $(5 \mathrm{~mm}$ diameter) from fungal colonies were inoculated on the center of fungicide amended (all SDHIs) and nonamended PDA plates. After 3 days of incubation, two perpendicular colony diameters were measured using 16EX digital calipers (Mahr). Each SDHI amended PDA colony diameter was divided by a nonamended PDA colony diameter and multiplied by 100 to calculate RMG\%. Two colony diameters were measured on each petri plate, and two petri plates were used in two repeated experiments (8 colony diameter measurements total). The repeated experiments were not significantly different, and all colony measurements were combined for statistical analysis.

For statistical analysis, S. homoeocarpa isolates were grouped into three categories based on target gene mutation patterns ( $S d h \mathrm{~B}$ H267Y, SdhC G91R, and no mutation on either of the genes) with a minimum of 5 isolates per group. Analysis of variance was conducted on target gene mutation groups, and Fisher's protected least significant difference (LSD) was conducted to compare RMG\% values among mutation groups for each fungicide using the JMP software package, version 10.0 (SAS Institute Inc.).

DNA extraction, polymerase chain reaction (PCR) amplification, and sequencing. DNA of 25 isolates was extracted using a CTAB method (Hulvey et al. 2012). Three PCR primer sets, F_ SdhB/R_SdhB, F_SdhC/R_SdhC, and F_SdhD/R_SdhD, were used to amplify the $S d h \mathrm{~B}, S d h \mathrm{C}$, and $S d h \mathrm{D}$ genes identified from a draft genome of $S$. homoeocarpa (Green et al. 2016). The PCR products of $S d h \mathrm{~B}, S d h \mathrm{C}$, and $S d h \mathrm{D}$ were amplified using Phusion taq polymerase (New England Biolabs), by touch-down PCR programs with different annealing temperatures at 72,68 , and $67^{\circ} \mathrm{C}$, respectively, decreasing at increments of $1.0^{\circ} \mathrm{C}$ per cycle for the first eight cycles and 64,60 , and $59^{\circ} \mathrm{C}$ of the remaining 45 cycles. A total $20 \mu \mathrm{l}$ of volumes includes 1x Phusion HF Buffer, 0.2 dNTPs, $0.4 \mu \mathrm{M}$ primers, $1 \mathrm{U}$ Phusion taq polymerase, $3 \%$ DMSO, and $<250 \mathrm{ng}$ of gDNA. Amplicons were purified using PureLink PCR Micro kit (Invitrogen). The purified amplicons were sequenced by Macrogen Corporation (Boston, MA).

Plasmid construction and generation of $S d h \mathrm{~B}$ or $S d h \mathrm{C}$ mutants from a SDHI-sensitive isolate. The 1,500-bp upstream region and full length of $S d h \mathrm{~B}$ (941 bp) was amplified from gDNA of isolate 5.1 harboring the $\mathrm{B}-\mathrm{H} 267 \mathrm{Y}$ mutation using primer F_AapI up1500SdhB and R_SacI_SdhB. The 1,500-bp upstream region and full length of $S \bar{d} h \mathrm{C}$ (759 bp) was also amplified from gRNA of isolate M1 harboring C-G91R mutation using primer F_ApaI_ up1500SdhC and R_NotI_SdhC. Each amplicon purified by Zymo Gel DNA recovery kit (Zymo research, Irvine, CA, USA) and plasmid pYHN3-MCS (Sang et al. 2017) was digested with ApaI/SacI for $S d h \mathrm{~B}$ gene or ApaI/NotI for $S d h \mathrm{C}$ gene. Two digested products were purified and ligated to generate the plasmid pYHN3-SdhB(H267Y) or pYHN3-SdhC(G91R). The plasmid DNA (5 $\mu \mathrm{g})$ was used for a polyethylene glycol (PEG)-mediated transformation in the protoplasts from isolate HRS10. The protoplast generation and PEGmediated transformation were conducted according to Sang et al. (2017). After 7 days, hygromycin resistant transformants were grown on $100 \mu \mathrm{g} \mathrm{ml}^{-1}$ of hygromycin amended regeneration agar medium (239.6 $\mathrm{g}$ of sucrose, $0.5 \mathrm{~g}$ of yeast extract, $15 \mathrm{~g}$ of agar per liter). Two S. homoeocarpa mutants $\mathrm{HRS} 10\left(\mathrm{SdhB}^{\mathrm{H} 267 \mathrm{Y}}\right)$ and $\mathrm{HRS} 10\left(\mathrm{SdhC}^{\mathrm{G} 91 \mathrm{R}}\right)$ and one control isolate HRS10 were used for in vitro sensitivity assays to five SDHI fungicides (boscalid, fluxapyroxad, isofetamid, penthiopyrad, and fluopyram). The diameter of fungal colonies from three individual plates was measured after the isolate, and mutants grew for $144 \mathrm{~h}$ on PDA amended with $1,000 \mu \mathrm{g} \mathrm{ml}^{-1}$ of boscalid, fluxapyroxad,

SdhB $(n=15) 251$ EERKAALDNS MSLYRCHTIL NCSRTCPKGL NPGLAIAEIK 280 $\operatorname{SdhB}(\mathrm{H} 267 \mathrm{r})(\mathrm{n}=10) 251$ EERKAALDNS MSLYRCYTIL NCSRTCPKGL NPGLAIAEIK 280

Fig. 1. Alignment of partial deduced amino acid sequences of $S d h B$ from 25 field isolates used in this study. " $n$ " indicates number of isolates. SdhB $(\mathrm{H} 267 \mathrm{Y})$ refers to substitution at amino acid position 267 (shaded letter) of SdhB gene in 10 isolates (5.1, 5.2, J-5, J-7, J-10, J-12, J-13, J-15, J-16, and J-17) from Japan and is correlated with resistance to boscalid and penthiopyrad.

Table 1. Primers used in this study

\begin{tabular}{llc}
\hline Primers name & \multicolumn{1}{c}{ Primer sequence $\left.\mathbf{( 5}^{\prime} \mathbf{- 3}^{\prime}\right)$} & Description \\
\hline F_SdhB & CGGCTCTCGTTTGCCATTGA & Amplification and sequencing primers for \\
R_SdhB & TACACAAGCCGCATTCGCAG & SdhB gene $(941$ bp $)$ \\
F_SdhC & CTTCCGCATCAACGACGATA & Amplification and sequencing primers for \\
R_SdhC & TCCTCTTGGGAGACCTCAT & SdhC gene $(759$ bp $)$ \\
F_SdhD & TGATGAGTAGCCGAGCTAC & Amplification and sequencing primers for \\
R_SdhD & CTGCTCACATAATCTCGCTTTC & SdhD gene $(683$ bp) \\
F_ApaI_up1500SdhB & ATGCGGGCCCAAACAACTCTATTCAGCCTC & Amplification of upstream region and SdhB \\
R_SacI_SdhB & ATGCGAGCTCTTAAAAAGCCATCTCCTTCTTGATCTCC & gene $(2,441$ bp $)$ \\
F_ApaI_up1500SdhC & ATTTGGGCCCGGTCCCACTGCAATTGAACC & Amplification of upstream region and SdhC \\
R_NotI_SdhC & ATTTGCGGCCGCCTAGAAGAACGCAACCAAAG & gene $(2,259$ bp $)$ \\
\hline
\end{tabular}


or isofetamid, and for $72 \mathrm{~h}$ on PDA amended with $1,000 \mu \mathrm{g} \mathrm{ml}^{-1}$ of penthiopyrad or fluopyram. The diameter of fungal colonies on PDA (control) plates was measured after $48 \mathrm{~h}$. Fisher's protected least significant difference (LSD) was conducted to compare diameter (mm) among three strains to each fungicide and control. The 1,500 bp of upstream region and full length of $S d h \mathrm{~B}, S d h \mathrm{C}$, and $S d h \mathrm{D}$ from HRS10 were deposited in GenBank with accession numbers MG708132, MG708133, and MG708134, respectively. All primers used in this study are listed in Table 1.

Prediction of protein structure and ligand binding sites of SdhB and SdhC. I-TASSER (Iterative Threading ASSEmbly Refinement) (https://zhanglab.ccmb.med.umich.edu) was used to predict protein structure and ligand binding sites $S d h \mathrm{~B}$ and $S d h \mathrm{C}$ in S. homoeocarpa (Roy et al. 2012; Yang and Zhang 2015; Zhang 2009). The full length of amino acid sequences of $S d h \mathrm{~B}$ and $S d h \mathrm{C}$ from draft genome sequences of isolate HRS10 (Green et al. 2016) were used for the prediction. PyMOL was used to generate the protein structure.

\section{Results}

Sequence analysis of $S d h B, S d h C$, and $S d h D$ genes. To find the genetic determinant conferring SDHI resistance in $S$. homoeocarpa, the SDHI target genes $(S d h \mathrm{~B}$ and $S d h \mathrm{C})$ from all 25 isolates were sequenced. Since no sequence differences in the $S d h \mathrm{D}$ gene were detected in 8 representative isolates, the rest of the isolates were not sequenced. Four single nucleotide changes that resulted in amino acid substitutions were detected (Figs. 1 and 2), and one nucleotide that caused a silent mutation was also detected. Ten Japanese isolates contained a mutation in $S d h \mathrm{~B}$ (CAC to TAC) at codon 267 that

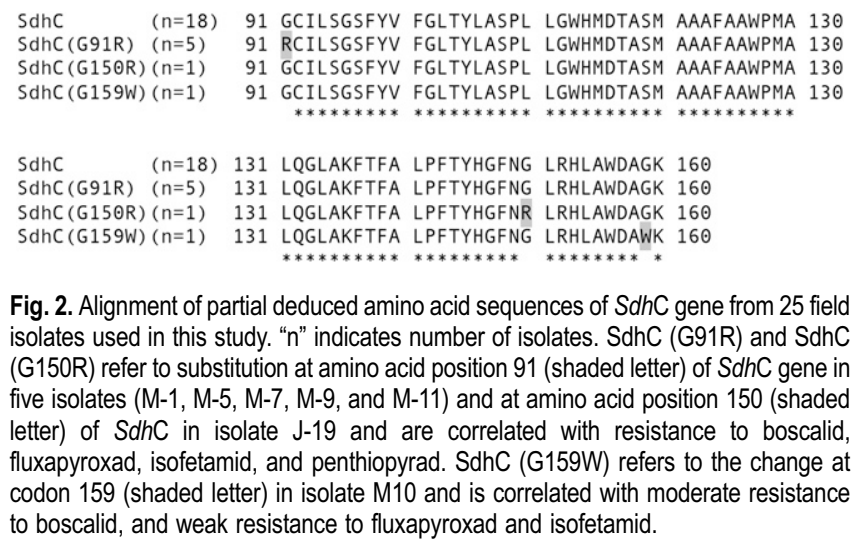

Fig. 2. Alignment of partial deduced amino acid sequences of $S d h C$ gene from 25 field isolates used in this study. "n" indicates number of isolates. SdhC (G91R) and SdhC (G150R) refer to substitution at amino acid position 91 (shaded letter) of SdhC gene in five isolates (M-1, M-5, M-7, M-9, and M-11) and at amino acid position 150 (shaded letter) of SdhC in isolate J-19 and are correlated with resistance to boscalid, fluxapyroxad, isofetamid, and penthiopyrad. SdhC (G159W) refers to the change at codon 159 (shaded letter) in isolate M10 and is correlated with moderate resistance to boscalid, and weak resistance to fluxapyroxad and isofetamid.

resulted in substitution of histidine by tyrosine (H267Y) (Fig. 1). Five Rhode Island isolates contained a mutation in $S d h$ C (GGA to CGA) at codon 91 that resulted in substitution of glycine by arginine (G91R) (Fig. 2). One Japanese isolate (J-19) contained a change in $S d h$ C (GGA to AGA) at codon 150 that resulted in substitution of glycine by arginine (G150R) (Fig. 2). One Rhode Island isolate (M-10) contained a change in $S d h$ C (GGG to TGG) at codon 159 that resulted in substitution of glycine by tryptophan (G159W) (Fig. 2). Isolates M-2, M-8, and M-10 contained a change in $S d h \mathrm{~B}$ (CTT to CTC) at codon 181 that did not result in an amino acid substitution; however, reduced SDHI in vitro sensitivity was observed in all three isolates.

S. homoeocarpa field isolates SDHI in vitro fungicide sensitivity. To investigate cross-resistance among SDHI fungicides in S. homoeocarpa, in vitro sensitivity assays of 25 field isolates to boscalid, fluopyram, fluxapyroxad, isofetamid, and penthiopyrad were conducted. Twenty-one isolates categorized into three groupings, C-G91R mutation $(n=5)$, B-H267Y mutation $(n=10)$, and the sensitive reference isolates (no mutation, $n=6$ ), were compared for mean RMG\% among SDHI active ingredient except for isolates J-19, M-2, M-8, and M-10. These isolates were excluded due to the small group size.

The C-G91R mutation group expressed significantly higher mean RMG\% values on all SDHI active ingredients than the B-H267Y mutation group and sensitive reference isolate/no mutation group (Table 2). The B-H267Y mutation group also expressed higher RMG\% values than the sensitive reference isolate/no mutation group on all SDHI active ingredients except fluopyram (Table 2). Increased sensitivity to fluopyram was observed from all isolates in the BH267Y mutation group only (Table 2). Isolate J-19 displayed a similar cross-resistance profile to isolates in the C-G91R mutation group, and isolate M-10 with both a silent mutation $181 \mathrm{C}>\mathrm{T}$ and C-G159W displayed a similar cross-resistance profile to isolates in the B-H267Y group (Table 2). Isolates M-2 and M-8 more closely resembled the C-G91R group for all active ingredients except fluxapyroxad.

Generation of SdhB ${ }^{\mathrm{H267Y}}$ and $\mathrm{SdhC}^{\mathrm{G91R}}$ mutants and fungicide sensitivity assay. A plasmid with the 1,500-bp of upstream and full length of $S d h \mathrm{~B}$ gene containing $\mathrm{H} 267 \mathrm{Y}$ or $S d h \mathrm{C}$ gene containing G91R was transformed into the SDHI sensitive isolate HRS10 to test if the H267Y mutation in $S d h$ B or G91R mutation in $S d h$ C confers SDHI resistance in S. homoeocarpa. The mutants $\mathrm{HRS} 10\left(\mathrm{SdhB}^{\mathrm{H} 267 \mathrm{Y}}\right)$ exhibited resistance to boscalid and penthiopyrad but not to fluxapyroxad, isofetamid, and fluopyram. The mutant HRS10(SdhC ${ }^{\mathrm{G} 91 \mathrm{R}}$ ) displayed resistance to boscalid, fluxapyroxad, penthiopyrad, and isofetamid but not to fluopyram. Furthermore, the

Table 2. The percentage (\%) of relative mycelium growth of 25 field Sclerotinia homoeocarpa isolates from Japan and the United States to five SDHI fungicides

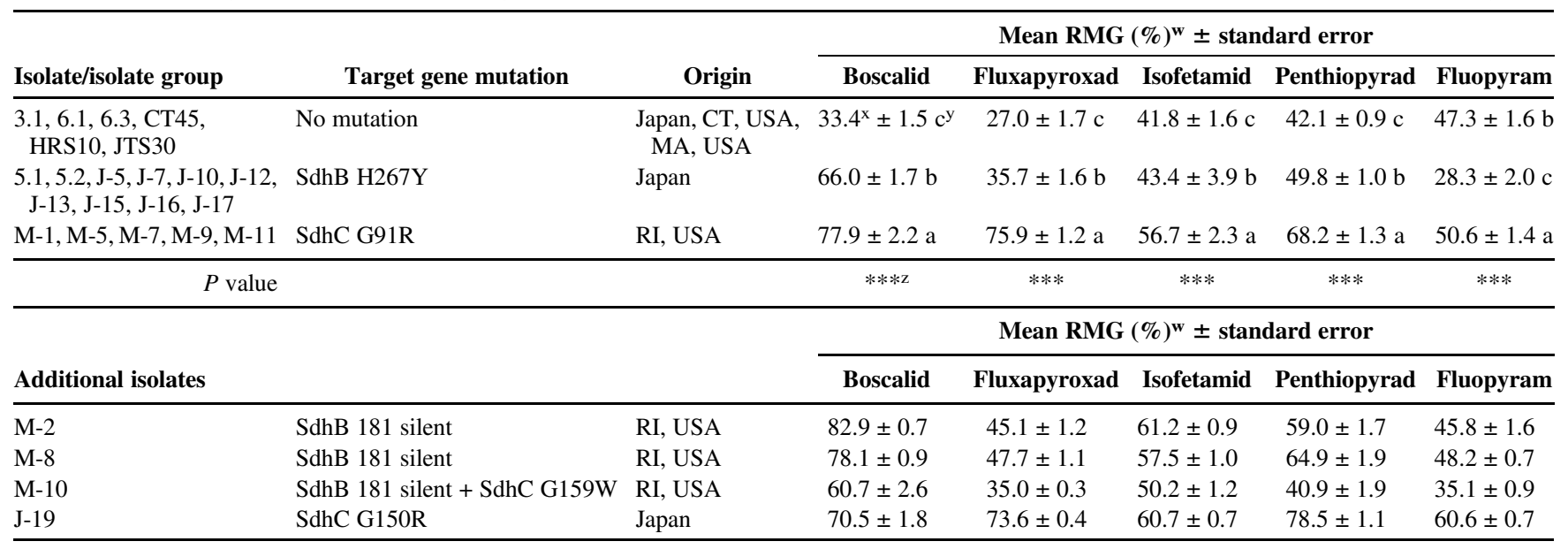

${ }^{w}$ Relative mycelial growth (RMG) (\%) was calculated by dividing the mean colony diameter of each isolate on PDA amended with each SDHI fungicide by the mean colony diameter of each isolate grown on nonamended PDA.

${ }^{x}$ Mean RMG\% is composed of the isolates in the group.

y Means followed by the same letter within each column are not significantly different according to Fisher's protected least significant difference test $(\alpha=0.05)$. $\mathrm{z} * * *=$ significance at $P \leq 0.001$. 
mutant HRS10(SdhC G91R) showed higher levels of resistance to boscalid and penthiopyrad than the mutant HRS10( $\left.\mathrm{SdhB}^{\mathrm{H} 267 \mathrm{Y}}\right)$ (Fig. 3).

Prediction of ligand binding sites in SdhB and SdhC. The protein structure and ligand binding sites of $S d h \mathrm{~B}$ and $S d h \mathrm{C}$ in
S. homoeocarpa were predicted. In the $S d h \mathrm{~B}$ subunit, predicted ligand binding sites were R220, S221, W224, H267, and I269. The mutated binding site (H267) was found in isolates 5.1, 5.2, J-5, J-7, J-10, J-12, $\mathrm{J}-13, \mathrm{~J}-15, \mathrm{~J}-16$, and $\mathrm{J}-17$. This protein was described as $3 \mathrm{vraF}$ in PDB (Protein Data Bank). In SdhC subunit, predicted sites were

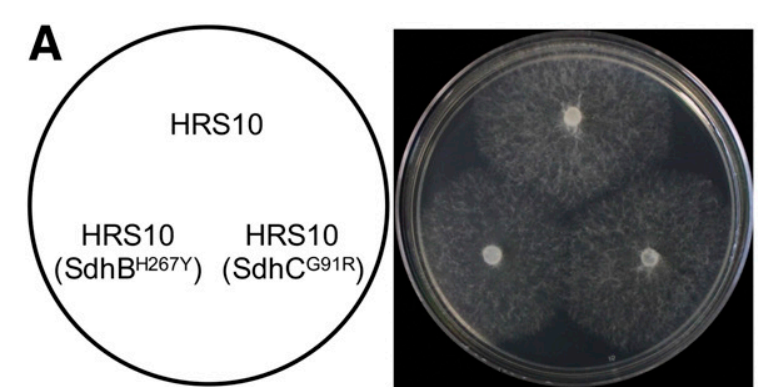

Control

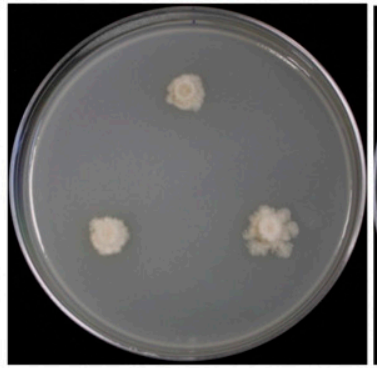

Fluxapyroxad $(1000 \mu \mathrm{g} / \mathrm{ml})$

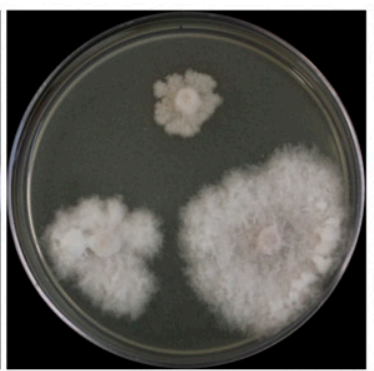

Boscalid $(1000 \mu \mathrm{g} / \mathrm{ml})$

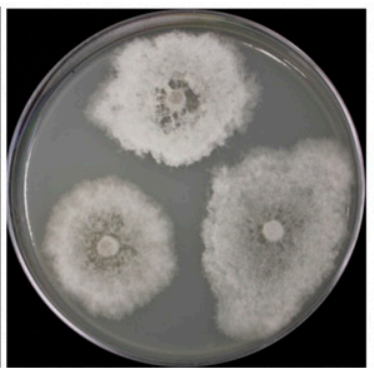

Isofetamid

$(1000 \mu \mathrm{g} / \mathrm{ml})$

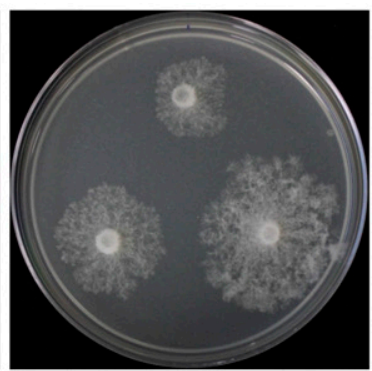

Penthiopyrad

$(1000 \mu \mathrm{g} / \mathrm{ml})$

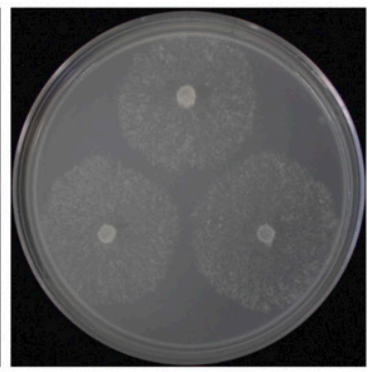

Fluopyram

$(1000 \mu \mathrm{g} / \mathrm{ml})$
B

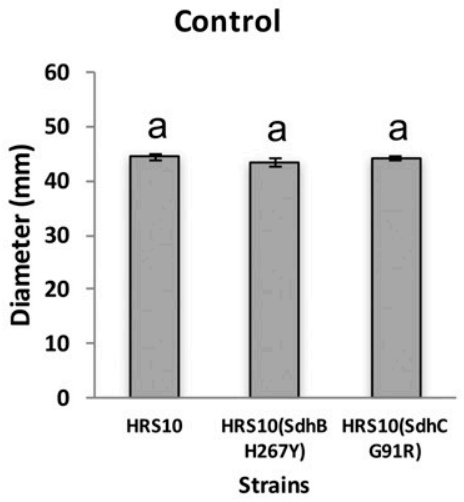

Fluxapyroxad $(1000 \mu \mathrm{g} / \mathrm{ml})$

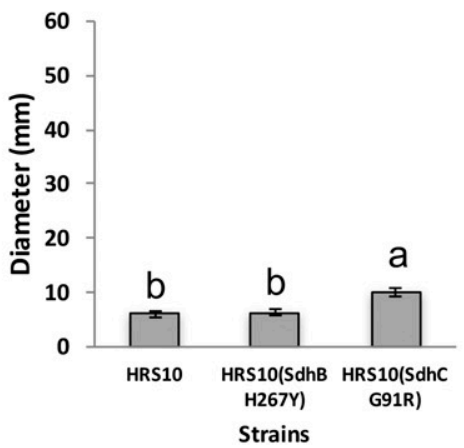

Boscalid $(1000 \mu \mathrm{g} / \mathrm{ml})$

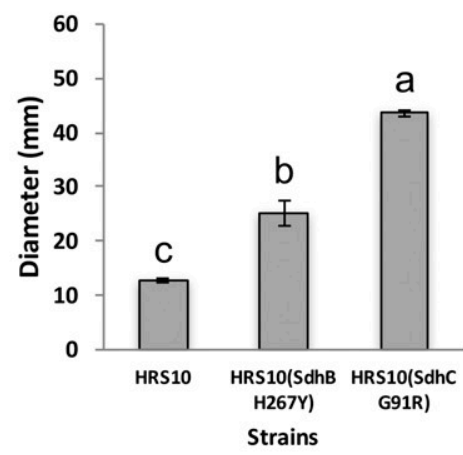

Isofetamid $(1000 \mu \mathrm{g} / \mathrm{ml})$

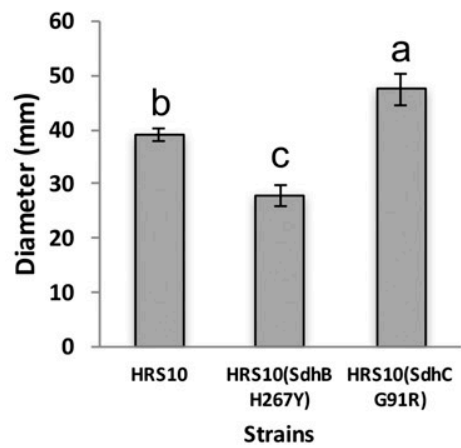

Penthiopyrad (1000 $\mu \mathrm{g} / \mathrm{ml})$

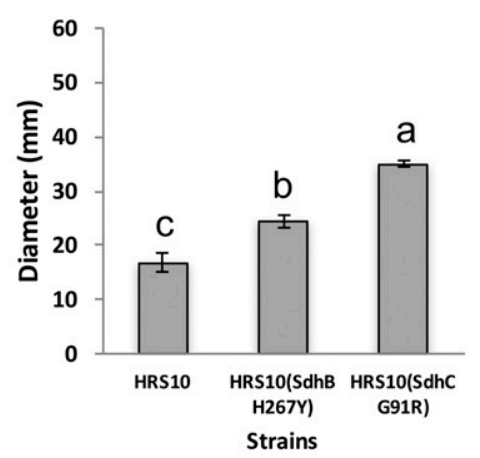

Fluopyram $(1000 \mu \mathrm{g} / \mathrm{ml})$

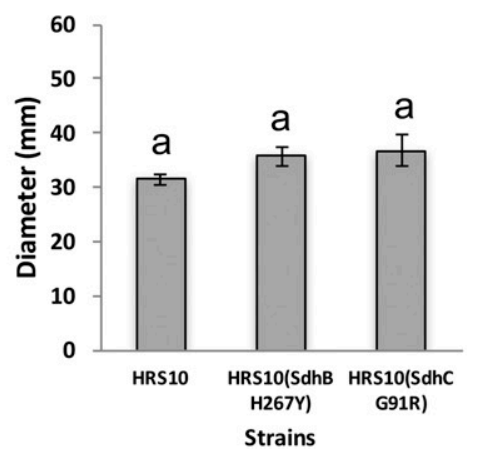

Fig. 3. Sensitivity of Sclerotinia homoeocarpa isolates HRS10 and mutants HRS10(SdhB ${ }^{\mathrm{H} 267 \mathrm{Y}}$ ) and $\mathrm{HRS10}\left(\mathrm{SdhC} \mathrm{C}^{\mathrm{G} 91 \mathrm{R}}\right.$ ) to five SDHI active ingredients (boscalid, penthiopyrad, fluxapyroxad, isofetamid, and fluopyram). (A) The pictures were taken after the isolate and mutants grew for $144 \mathrm{~h}$ on PDA amended with $1,000 \mu \mathrm{g} \mathrm{ml}^{-1}$ of boscalid, fluxapyroxad, and isofetamid, and for $72 \mathrm{~h}$ on PDA amended with $1,000 \mathrm{\mu g} \mathrm{ml}^{-1}$ of penthiopyrad and fluopyram. The picture of control plate without treatments was taken after $48 \mathrm{~h}$. (B) Diameter $(\mathrm{mm})$ of fungal colonies on PDA without (control) and amended with SDHI fungicides. Means followed by the same letter are not significantly different according to Fisher's protected least significant difference test $(\alpha=0.05)$. 
N87, R88, G91, L94, S95, H146, G147, G150, and H153. The binding site G91 mutated was found in isolates M-1, M-5, M-7, M-9, and $\mathrm{M}-11$, and the binding site G150 was mutated in isolate J-19. This protein was described as $3 \mathrm{ae} 3 \mathrm{C}$ in PDB (Fig. 4).

\section{Discussion}

The current study reports the occurrence of $S$. homoeocarpa field isolates exhibiting differential resistance to SDHI fungicides for the first time and elucidates the molecular mechanisms of SDHI resistance in $S$. homoeocarpa. We observed five different mutations in the $S d h \mathrm{~B}(\mathrm{H} 267 \mathrm{Y}$ and a silent mutation $181 \mathrm{C}>\mathrm{T})$ and $S d h \mathrm{C}$ (G91R, G150R, and G159W) subunit genes in S. homoeocarpa isolates from two golf courses. The B-H267Y mutation was determined to be a direct factor in resistance to boscalid and penthiopyrad, and the $\mathrm{C}-\mathrm{G} 91 \mathrm{R}$ was confirmed to be a direct factor conferring resistance to boscalid, fluxapyroxad, isofetamid, and penthiopyrad through the genetic transformation system. In addition, isolate J-19 displayed resistance to boscalid, fluxapyroxad, isofetamid, and penthiopyrad, which contained the C-G150R mutation. Isolate M-10 contained double mutations (C-G159W and a silent mutation $181 \mathrm{C}>\mathrm{T}$ in $S d h \mathrm{~B}$ ), and displayed moderate resistance to boscalid, and weak resistance to fluxapyroxad and isofetamid. Lastly, two isolates (M-2, M-8) with the silent mutation $S d h \mathrm{~B} 181 \mathrm{C}>\mathrm{T}$ displayed resistance to boscalid, fluxapyroxad, isofetamid, and penthiopyrad. The presence of multiple target gene mutations causing differential cross-resistance in $S$. homoeocarpa field isolates from two separate continents is cause for concern.

In total, 16 different plant-pathogenic fungal species have reported to be resistant to SDHI fungicides due to mutations in three of four SDH subunit genes (FRAC 2017b). Reports of SDHI resistance in other frequently treated plant pathogens have led to heightened alertness for SDHI resistance in S. homoeocarpa due to the over-reliance on fungicides for dollar spot control in turfgrass (Sierotzki and Scalliet 2013). Location and climatic conditions greatly influences disease occurrence, and 3 to 10 applications per year are commonly made to control dollar spot. Fungicide rotations/mixtures are an essential component of successful resistance management strategies because resistance to the benzimidazole and DMI classes are commonly found along with occasional resistance to the dicarboximide class (Putman et al. 2010; Sang et al. 2017). The current status of fungicides that effectively control dollar spot is fairly limited, and therefore delaying the onset of resistance to the SDHI class is critical. Differences in the SDHI cross-resistance profile of the isolates examined in this study suggest that fluopyram may be effective on populations that harbor the target gene mutations presented in this work; however, many other target gene mutations exist in other plant pathogens and may develop in $S$. homoeocarpa populations later (FRAC 2015; Sierotzki and Scalliet 2013). Several studies have reported high percentages of boscalid resistance in the absence of fluopyram resistance (Avenot et al. 2012; De Miccolis Angelini et al. 2014; Hu et al. 2016; Ishii et al. 2011; Veloukas et al. 2013).

SDHI field resistant isolates harboring the B-H267Y mutation and $\mathrm{SdhB}^{\mathrm{H} 267 \mathrm{Y}}$ mutants showed resistance to boscalid and penthiopyrad, increased sensitivity to fluopyram, and no resistance to fluxapyroxad and isofetamid (Table 2; Fig. 3). The histidine at codon 267 is a highly conserved residue, which is associated with the (3Fe-4 S) high-potential nonheme iron sulfur-redox (S3) center (Skinner et al. 1998). Horsefield et al. (2006) suggested the conserved histidine residue of $S d h \mathrm{~B}$ might strongly interact with the histidine and serine residue at codon 27 of $S d h \mathrm{C}$, blocking ubiquinone from accessing the quinone-binding site (Q-site) based on the structural and computational analysis of Q-site from Escherichia coli using carboxin. Therefore, the histidine residue was suggested to be essential for carboximide binding (Avenot et al. 2008), and the mutation in this residue might affect direct fungicide binding. The mutation from the conserved histidine residue to tyrosine in $S d h \mathrm{~B}$ gene was also reported in SDHI field resistant isolates of other plant-pathogenic fungi, such as Alternaria alternata, Botrytis cinerea, Corynespora cassiicola, and Pyrenophora teres (Avenot et al. 2008; Miyamoto et al. 2010; Stammler et al. 2014; Veloukas et al. 2013; Yin et al. 2011).
The increased sensitivity response to fluopyram in isolates containing the $S d h \mathrm{~B}$ H267Y mutation may be due to fluopyram possessing a benzamide ring that is better equipped to bind to the Q-pocket of resistant isolates containing the mutation (histidine to tyrosine) (Veloukas et al. 2013). Other fungal species with the same substitution also displayed increased sensitivity to benzamide fungicides (Avenot et al. 2012; Ishii et al. 2011; Scalliet et al. 2012; Shima et al. 2011; Veloukas et al. 2013). However, increased sensitivity to fluopyram was not confirmed by the $\mathrm{SdhB}^{\mathrm{H} 267 \mathrm{Y}}$ mutants of $S$. homoeocarpa due to possession of both wild and mutated SdhB genes in the mutant cells. In the presence of fluopyram, the wild SdhB gene might be dominant in the fungal cells, and this may explain why mutants had the same growth rate as the wild-type isolate. Results from the genetic transformation assay suggest that despite statistical differences from the in vitro fungicide assay, mean RMG\% differences of $\sim 10 \%$ on fluxapyroxad and isofetamid amended media did not correlate to a true resistance response (Table 2; Fig. 3).

SDHI field resistant isolates containing the C-G91R mutation and SdhC ${ }^{\mathrm{G} 91 \mathrm{R}}$ mutants modified from the sensitive isolate showed high resistance to boscalid, fluxapyroxad, and penthiopyrad, moderate resistance to isofetamid, and no resistance to fluopyram (Table 2). According to the FRAC SDHI working group meeting in 2016, the C-G79R mutation in P. teres confers moderate resistance in the field, and this mutation was first detected in 2013 in Europe. This position is homologous to the mutation present in the five resistant isolates from Rhode Island (M-1, M-5, M-7, M-9, and M-11) and represents the first report of a mutation in this position in North America. In $S$. homoeocarpa, the C-G91R mutation exhibited the highest resistance compared with the SDHI-sensitive isolate group and the widest

A

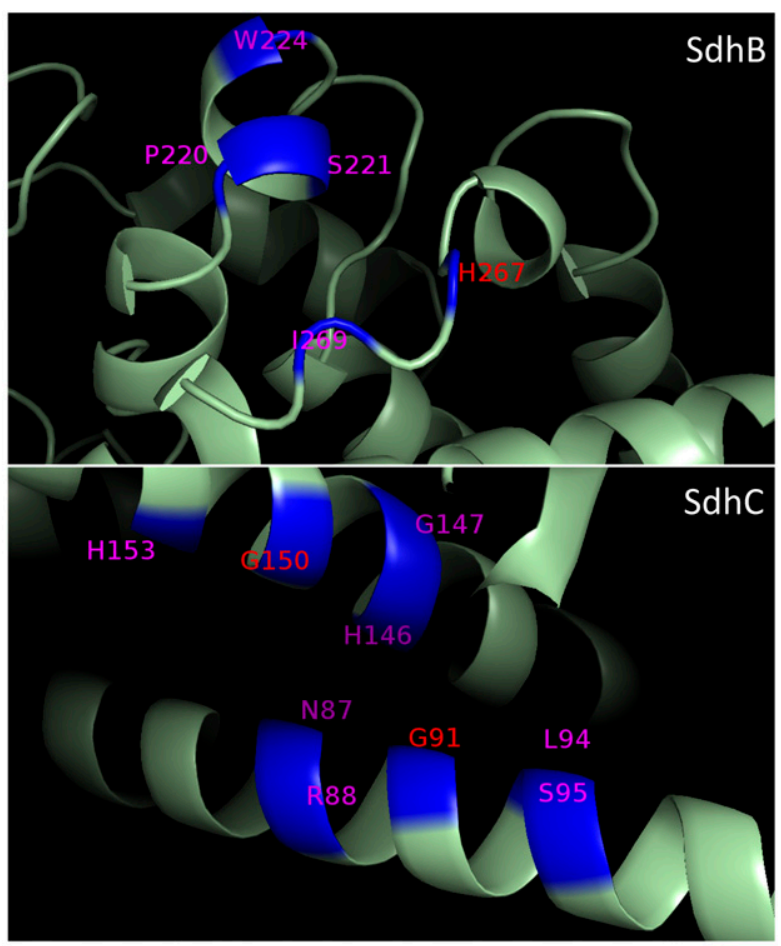

B

\begin{tabular}{|l|l|l|}
\hline $\begin{array}{l}\text { Subunit } \\
\text { Name }\end{array}$ & Predicted Ligand Binding Site & Mutation Found \\
\hline SdhB & $\begin{array}{l}\text { R220, S221, W224, H267, } \\
\text { I269 }\end{array}$ & H267Y \\
\hline SdhC & $\begin{array}{l}\text { N87, R88, G91, L94, S95, } \\
\text { H146, G147, G150, H153 }\end{array}$ & G91R, G150R \\
\hline
\end{tabular}

Fig. 4. Ligand binding sites prediction of SdhB and SdhC from Sclerotinia homoeocarpa isolate HRS10. (A) Protein structure and ligand binding sites of SdhB and SdhC. (B) The lists of predicted ligand binding sites of SdhB and SdhC and mutations found in this study. 
spectrum of resistance across active ingredients (boscalid, fluxapyroxad, isofetamid, and penthiopyrad) in the class (Table 2). Furthermore, the active ingredients most-severely affected are pyrazole-4carboximide (fluxapyroxad and penthiopyrad) or pyridine-carboximide (boscalid) fungicides. Lastly, HRS10(SdhC ${ }^{\text {G91R }}$ ) mutants grew more on isofetamid-amended media than the HRS $10\left(\mathrm{SdhB}^{\mathrm{H} 267 \mathrm{Y}}\right)$ mutants and HRS10 and suggest the $15 \%$ increase in RMG\% observed from the in vitro fungicide assay correlates to a true resistance response.

Isolate J-19 was the only isolate determined to have the C-G150R mutation and resulted in a very similar cross-resistance profile to the C-G91R mutation (Table 2). There are no previous reports of a mutation in this position of the $S d h \mathrm{C}$ gene in any other fungal species, and this appears to be the first report. The predicted binding site simulation confirms that a mutation at this position is likely to alter SDHI fungicide binding affinity. Isolate M-10 was the only isolate determined to have the C-G159W mutation and showed high resistance to boscalid and increased fluopyram sensitivity as the B-H267Y mutation isolates (Table 2). Moreover, isolate M-10 exhibited weak or no resistance to fluxapyroxad, isofetamid, or penthiopyrad (Table 2). In addition, isolates M-2, M-8, and M-10 were discovered to have a silent mutation in $S d h \mathrm{~B}$ (CTT to CTC) at codon 181 that did not result in an amino acid substitution. Isolates M-2 and M-8 both showed resistance to boscalid, isofetamid, and penthiopyrad, but no resistance to fluopyram. Silent mutation has not been reported to cause SDHI resistance in any other fungal pathogens, and this is the first report. KimchiSarfaty et al. (2007) suggested that a silent mutation in nucleotide triplets can result in a different three-dimensional structure of the protein due to speed-dependent folding of an amino acid chain, influenced by slow processing of ribosomes. Also, Yamashita and Fraaije (2018) observed Zymoseptoria tritici isolates collected from the field that were resistant to isofetamid and fluopyram, but did not contain target gene mutations within the $S d h \mathrm{~B}, \mathrm{C}$, or D subunits. The function of the silent mutation in $S$. homoeocarpa should be validated using a genetic transformation system.

Sang et al. (2015) described that multidrug resistant isolates of $S$. homoeocarpa exhibited reduced sensitivity to propiconazole (DMI), iprodione (dicarboximide), and boscalid. These isolates harbor overexpression of an $\mathrm{ABC}$ efflux transporter ShPDRl, which is involved in decreased sensitivity to these different fungicide classes and was confirmed by a heterologous expression system in yeast (Sang et al. 2015). The field study of Allen-Perkins et al. (2017) also indicated that applications of boscalid selected DMI insensitive S. homoeocarpa isolates that overexpressed ShPDRl and supported existing evidence that $\mathrm{ABC}$-transporters are involved in resistance to chemically unrelated fungicides. Future research in field conditions is needed to determine the impact of qualitative (target gene mutations) and quantitative (gene overexpression) resistance on $S$. homoeocarpa population sensitivity in relation to SDHI fungicides.

Detection of differential resistance to SDHIs signals that turfgrass industry must implement sound fungicide resistance management strategies to prolong the longevity of this important fungicide class in S. homoeocarpa. S. homoeocarpa field isolates have already developed resistance to four different classes of single-site fungicides, multiple fungicide resistance, and multidrug resistance conferred by xenobiotic detoxification (Jo et al. 2006; Putman et al. 2010; Sang et al. 2017). Future research should focus on developing an integrated pest management approach by implementing effective cultural practices, weather-based disease forecasting, and initiating a longterm monitoring of resistant populations using molecular diagnostic tools. Furthermore, it is essential for academic and industry scientists to develop a better understanding of the mechanisms of differential resistance to SDHIs and management of field populations with multiple fungicide resistance. Lastly, recommendations for dollar spot control must emphasize rotation of different fungicide classes with single-site modes of action. Furthermore, multisite fungicides (chlorothalonil [FRAC \#M5] and fluazinam [FRAC \#29]) should be used as tank-mix options with single-site mode of action fungicides under periods of intense disease pressure. Hopefully, the sum of these practices can delay fungicide resistance to the SDHI fungicide class.

\section{Acknowledgments}

We thank golf course superintendents in Japan for sending dollar spot samples. The contents are solely the responsibility of the authors and do not necessarily represent the official views of the USDA or NIFA.

\section{Literature Cited}

Allan-Perkins, E., Campbell-Nelson, K., Popko, J. T., Sang, H., and Jung, G. 2017. Investigating selection of demethylation inhibitor fungicide-insensitive Sclerotinia homoeocarpa isolates by boscalid, flurprimidol, and paclobutrazol. Crop Sci. 57 (Suppl. 1):S-301-S-309.

Anthony, A., and Kerns, J. 2017. SDHI resistance screening in Sclerotinia homoeocarpa. (Abstr.) ASA-CSSA-SSSA Annual Joint Meeting. Tampa, FL, USA

Avenot, H. F., Sellam, A., Karaoglanidis, G., and Michailides, T. J. 2008 Characterization of mutations in the iron-sulphur subunit of succinate dehydrogenase correlating with boscalid resistance in Alternaria alternata from California pistachio. Phytopathology 98:736-742.

Avenot, H. F., Thomas, A., Gitaitis, R. D., Langston, D. B., Jr., and Stevenson, K. L. 2012. Molecular characterization of boscalid- and penthiopyrad-resistant isolates of Didymella bryoniae and assessment of their sensitivity to fluopyram. Pest Manag. Sci. 68:645-651.

Cole, H., Taylor, B., and Duich, J. 1968. Evidence of differing tolerances to fungicides among isolates of Sclerotinia homoeocarpa. Phytopathology 58: 683-686.

De Miccolis Angelini, R. M., Masiello, M., Rotolo, C., Pollastro, S., and Faretra, F. 2014. Molecular characterisation and detection of resistance to succinate dehyrogenase inhibitor fungicides in Botryotinia fuckeliana (Botrytis cinerea). Pest Manag. Sci. 70:1884-1893.

Detweiler, A. R., Vargas, J. M., and Danneberger, T. K. 1983. Resistance of Sclerotinia homoeocarpa to iprodione and benomyl. Plant Dis. 67:627-630.

Fungicide Resistance Action Committee. 2015. List of species resistant to SDHIs http://www.frac.info/working-group/sdhi-fungicides.

Fungicide Resistance Action Committee. 2017a. FRAC list of fungicide common names. http://www.frac.info/publications/downloads.

Fungicide Resistance Action Committee. 2017b. FRAC list of plant pathogenic organisms resistant to disease control agents. http://www.frac.info/publications/ downloads.

Gilstrap, D. M., and Vargas, J. M. 2005. Don't panic with Emerald. Proc. $75^{\text {th }}$ Annu. Michigan Turf. Conf. 75:1-6.

Golembiewski, R. C., Vargas, J. M., Jones, A. L., and Detweiler, A. R. 1995 Detection of demethylation inhibitor (DMI) resistance in Sclerotinia homoeocarpa populations. Plant Dis. 79:491-493.

Green, R., Sang, H., Chang, T., Allan-Perkins, E., Petit, E., and Jung, G. 2016 Draft genome sequences of the turfgrass pathogen Sclerotinia homoeocarpa. Genome Announc. 4:e01715-15.

Horsefield, R., Yankovskaya, V., Sexton, G., Whittingham, W., Shiomi, K., Omura, S., Byrne, B., Cecchini, G., and Iwata, S. 2006. Structural and computational analysis of the quinone-binding site of complex II (succinate ubiquinone oxidoreductase) - a mechanism of electron transfer and proton conduction during ubiquinone reduction. J. Biol. Chem. 281:7309-7316.

Hu, M.-J., Fernandez-Ortuño, D., and Schnabel, G. 2016. Monitoring resistance to SDHI fungicides in Botrytis cinerea from strawberry fields. Plant Dis. 100: 959-965.

Hulvey, J., Popko, J. T., Sang, H., Berg, A., and Jung, G. 2012. Overexpression of ShCYP51B and ShatrD in Sclerotinia homoeocarpa isolates exhibiting practical field resistance to a demethylation inhibitor fungicide. Appl. Environ. Microbiol. 78:6674-6682.

Ishii, H., Miyamoto, T., Ushio, S., and Kakishima, M. 2011. Lack of cross resistance to a novel succinate dehydrogenase inhibitor, fluopyram, in highly boscalid-resistant isolates of Corynespora cassiicola and Podosphaera xanthii. Pest Manag. Sci. 67:474-482.

Jo, Y., Niver, A., Rimelspach, J., and Boehm, M. J. 2006. Fungicide sensitivity of Sclerotinia homoeocarpa from golf courses in Ohio. Plant Dis. 90:807-813.

Kimchi-Sarfaty, C., Mi Oh, J., Kim, I. W., Sauna, Z. E., Calcagno, A. M., Ambudkar, S. V., and Gottesman, M. M. 2007. A "Silent" polymorphism in the MDR1 gene changes substrate specificity. Science 315:525-528.

Matsson, M., and Hederstedt, L. 2001. The carboxin-binding site on Paracoccus denitrificans succinate:quinone reductase identified by mutations. J. Bioenerg. Biomembr. 33:99-105.

Miyamoto, T., Ishii, H., Stammler, G., Koch, A., Ogawara, T., Tomita, T., Fountaine, J., Ushio, T., Seko, T., and Kobori, S. 2010. Distribution and molecular characterization of Corynespora cassiicola isolates resistant to boscalid. Plant Pathol. 59:873-881.

Putman, A. I., Jung, G., and Kaminski, J. E. 2010. Geographic distribution of fungicide-insensitive Sclerotinia homoeocarpa isolates from golf courses in the northeastern United States. Plant Dis. 94:186-195.

Roy, A., Yang, J., and Zhang, Y. 2012. COFACTOR: An accurate comparative algorithm for structure-based protein function annotation. Nucleic Acids Res. 40 (W1):W471-W477.

Sang, H., Hulvey, J., Popko, J. T., Lopes, J., Swaminathan, A., Chang, T., and Jung, G. 2015. A pleiotropic drug resistance transporter is involved in reduced sensitivity to multiple fungicide classes in Sclerotinia homoeocarpa. Mol. Plant Pathol. 16:251-261. 
Sang, H., Popko, J. T., Jr., Chang, T., and Jung, G. 2017. Molecular mechanisms involved in qualitative and quantitative resistance to the dicarboximide fungicide iprodione in Sclerotinia homoeocarpa field isolates. Phytopathology 107:198-207.

Scalliet, G., Bowler, J., Luksch, T., Kirchhofer-Allan, L., Steinhauer, D., Ward, K., Niklaus, M., Verras, A., Csukai, M., Daina, A., and Fonne-Pfister, R. 2012. Mutagenesis and functional studies with succinate dehydrogenase inhibitors in the wheat pathogen Mycosphaerella graminicola. PLoS One 7:e35429.

Shima, Y., Ito, Y., Hatabayashi, H., Koma, A., and Yabe, K. 2011. Five carboxinresistant mutants exhibited various responses to carboxin and related fungicides. Biosci. Biotechnol. Biochem. 75:181-184.

Sierotzki, H., and Scalliet, G. 2013. A review of current knowledge of resistance aspects for the next-generation succinate dehydrogenase inhibitor fungicides. Phytopathology 103:880-887.

Skinner, W., Bailey, A., Renwick, A., Keon, J., Gurr, S., and Hargreaves, J. 1998. A single amino acid substitution in the iron-sulphur protein subunit of succinate dehydrogenase determines resistance to carboxin in Mycosphaerella graminicola. Curr. Genet. 34:393-398.

Smiley, R. W., Dernoeden, P. H., and Clarke, B. B. 2005. Compendium of Turfgrass Diseases, 3rd Ed. American Phytopathological Society, St. Paul, MN.
Stammler, G., Rehfus, A., Prochnow, J., Bryson, R., and Strobel, D. 2014. New findings on the development of insensitive isolates of Pyrenophora teres towards SDHI fungicides. Julius-Kühn-Arch. 447:568.

Veloukas, T., Markoglou, A. N., and Karaoglanidis, G. S. 2013. Differential effect of $S d h \mathrm{~B}$ gene mutations on the sensitivity to SDHI fungicides in Botrytis cinerea. Plant Dis. 97:118-122.

Vincelli, P., Clarke, B., and Munshaw, G. 2017. Chemical control of turfgrass diseases 2017. Online Coop. Ext. Ser. Publ. No. PPA-1. University of Kentucky, Lexington, KY.

Yamashita, M., and Fraaije, B. 2018. Non-target site SDHI resistance is present as standing genetic variation in field populations of Zymiseptoria tritici. Pest Manage. Sci. 74:672-681.

Yang, J., and Zhang, Y. 2015. I-TASSER server: New development for protein structure and function predictions. Nucleic Acids Res. 43 (W1):W174-W181.

Yin, Y. N., Kim, Y. K., and Xiao, C. L. 2011. Molecular characterization of boscalid resistance in field isolates of Botrytis cinerea from apple. Phytopathology 101:986-995.

Zhang, Y. 2009. I-TASSER: Fully automated protein structure prediction in CASP8. Proteins 77, S9, Suppl. 9:100-113. 